\title{
Escarabajos coprófagos (Coleoptera: Scarabaeinae) asociados a excrementos de mamíferos en un fragmento de bosque seco tropical en el Departamento del Atlántico, Colombia
}

\author{
Neis Martínez Hernandez ; Greisha Salcedo Muñoz²; Katherine Sierra Quintero² \& \\ JeISON BARRAZA MÉNDEZ ${ }^{1,3, \bigotimes}$
}

1. Grupo de investigación Biodiversidad del Caribe colombiano. Facultad de Ciencias Básicas. Universidad del Atlántico, Colombia. 2. Universidad del Atlántico, Colombia. 3. Centro de Ecología. Instituto Venezolano de Investigaciones Científicas (IVIC). Caracas, República Bolivariana de Venezuela.

\begin{abstract}
Resumen. La expansión agrícolo-ganadera puede afectar de manera indirecta a la fauna nativa. Debido a esto se evaluó la composición y la estructura de escarabajos coprófagos asociados a excrementos de Alouatta seniculus, Sus domestica y Bos taurus en un fragmento de bosque seco tropical en la Reserva Campesina Tierra Arena (RCTA), Atlántico. Se realizaron cuatro muestreos entre junio y octubre de 2008. Se marcaron 10 puntos distanciados $50 \mathrm{~m}$ y en cada uno se colocaron en posición triangular tres trampas de caída, cebadas por separado con excremento de los mamíferos mencionados. Las trampas fueron revisadas a las 24 horas. Se recolectaron 864 individuos agrupados en 21 especies y nueve géneros, destacándose Uroxys sp. con $33.21 \%$ del total de individuos capturados. El mayor número de especies (18) e individuos (499) se capturó con las trampas cebadas con excremento de $A$. seniculus. La estructura de la comunidad de Scarabaeinae varió significativamente entre los diferentes tipos de cebo. El índice de Manly sugirió que la mayoría de las especies (13) prefieren el excremento de $A$. seniculus. La reducción de las poblaciones de de este primate debido a la expansión agrícologanadera podrían afectar de forma negativa a las poblaciones de Scarabaeinae que manifiestan afinidad por su excremento.
\end{abstract}

[Palabras clave: preferencia, Alouatta seniculus, Uroxys sp., ANOSIM, índice de Manly]

\begin{abstract}
Aвstract. Dung beetles (Coleoptera: Scarabaeinae) associated to mammalians feces in a tropical dry forest fragment in Department of Atlántico, Colombia: The composition and structure of the dung beetles associated with the dung of the Alouatta seniculus, Sus domestica and Bos Taurus in a fragment of a dry tropical forest in the Reserva Campesina Tierra Arena (RCTA), Atlántico, was evaluated. Four samples since June to October of 2008 was made. It was chosen ten points separated in $50 \mathrm{~m}$ each other. On every point was placed three pitfalls traps in a triangle position, every trap was baited with a different dung of the mammal named and checked within 24 hours. We captured 864 individuals grouped in nine genera and 21 species. Uroxys sp. was the most abundant species with $33.21 \%$ of the total of individuals captured. The bait with most diverse (18) and abundance (499) was the monkey's dung. The ANOSIM test showed differences in structure the study area $(\mathrm{R}=0.422 ; p=0.001)$. The Manly's index showed that most of the Scarabaeinae species (13) had preference for monkey's dung, these results showed that dung beetles had preference for dung of native mammals, such as the howler monkey (A. seniculus), whose habitat reduction and the human activities may cause also the fauna associated to this animal like the dung beetles.
\end{abstract}

[Keywords: preferences, Alouatta seniculus, Uroxys sp., ANOSIM]

\section{INTRODUCCIÓN}

En los últimos años, la expansión de las fronteras agrícolas y ganaderas en las tierras bajas del Caribe colombiano ha provocado una disminución en la extensión de ecosistemas naturales, como el bosque seco tropical (BsT) (IAvH 1998). La extracción de madera y la fragmentación del Bs-T alteran de manera directalas condiciones ambientales y modifican la estructura de las comunidades biológicas,

Centro de Ecología. Instituto Venezolano de Investigaciones Científicas(IVIC).CarreteraPanamericana, $\mathrm{km} 11$. Altos de Pipe. Apartado postal 21827. Caracas 1020A. República Bolivariana de Venezuela. jeisonbarrazamendez@gmail.com tales como los mamíferos. Estas alteraciones, a su vez, pueden afectar indirectamente otros ensambles de animales, como los escarabajos coprófagos. En particular, las variaciones en la abundancia o composición de las comunidades de mamíferos pueden afectar al gremio trófico de los escarabajos coprófagos de la subfamilia Scarabaeinae (Medina et al. 2001) debido a que se encuentran asociados de manera muy estrecha a estos vertebrados por alimentarse de sus heces (Halffter \& Edmonds

Editor asociado: Alejandro Farji-Brener

Recibido: 2 de septiembre de 2011; Fin de arbitraje: 19 de diciembre; Revisión recibida: 25 de abril de 2012; Aceptado: 3 de mayo. 
1982). Diferentes estudios apoyan esta idea al sugerir que los recursos para los escarabajos coprófagos en los bosques tropicales son limitados, lo cual incrementa la competencia por el excremento (Halffter \& Edmonds 1982; Hanski 1991) y genera una relación muy fuerte con los mamíferos nativos, en especial con los primates (Andresen 2005).

Diversos trabajos demuestran la relación estrecha que existe entre los coleópteros coprófagos y la fauna de primates neotropicales (Gill 1991; Estrada et al. 1993; Castellanos et al. 1999). Sin embargo, la mayoría de estos estudios se han realizado en zonas diferentes al Bs-T. Además, la mayoría de las investigaciones emplean como atrayente heces humanas (Escobar et al. 2005; Bohórquez \& Montoya 2009; Barraza et al. 2010). Por lo tanto, la información de la relación entre primates u otros mamíferos con los Scarabaeinae en el Bs-T es escasa. En consecuencia, para comprender el efecto del avance de la frontera agrícolo-ganadera sobre estos insectos es muy importante determinar cómo varía el ensamble de escarabajos coprófagos cuando los excrementos de mamíferos nativos (i.e., primates) son reemplazados por los producidos por mamíferos exóticos (i.e., ganado). En este trabajo se evaluó la composición y estructura de Scarabaeinae asociados a excrementos de $A$. seniculus Linnaeus 1766, S. domestica Linnaeus 1758 y $B$. taurus Linnaeus 1758 en un fragmento de Bs-T, en la Reserva Campesina de Tierra Arena (RCTA), Departamento del Atlántico, Colombia.

\section{MÉTOdOS}

\section{Área de estudio}

La reserva campesina de Tierra Arena (RCTA) fue declarada en categoría de reserva campesina en 1996 (Decreto 1777) y se encuentra localizada en el municipio de Juan de Acosta, Departamento del Atlántico, Colombia. La zona está ubicada a $10^{\circ} 46^{\prime} 02.6^{\prime \prime} \mathrm{Ny} 75^{\circ} 0.2^{\prime} 34^{\prime \prime} \mathrm{O}$, a una altitud que oscila entre 160 y 250 m.s.n.m. y posee una extensión de $\sim 47$ ha. Los suelos del bosque son arcilloso-arenosos (ArA), permeables, con alta cantidad de materia orgánica. La reserva se encuentra en la región biogeográfica del Cinturón Árido Pericaribeño (Hernández et al. 1991) y constituye una formación higrotropofítica según la clasificación de Holdridge et al. (1971), correspondiente a un bosque seco tropical (Bs-T), con $28^{\circ} \mathrm{C}$ de temperatura anual promedio. El régimen pluviométrico promedio en la zona es de $179 \mathrm{~mm} /$ año. Las lluvias son de régimen bimodal, con dos picos a lo largo del año, entre los meses de abril a junio y de septiembre a noviembre. Los valores de humedad relativa presentan una mayor inestabilidad atmosférica en los períodos abril-mayo y agosto-noviembre, con un rango promedio entre $79 \%$ y $82 \%$ anual (Instituto de Hidrología, Meteorología y Estudios Ambientales de Colombia, IDEAM).

En la reserva es frecuente observar mamíferos nativos como Agouti paca, Dasyprocta punctata, Sylvilagus oridanus, Coendú prehensils y A. seniculus (mono aullador), mientras que en su periferia se encuentran fincas y parcelas dedicadas a cultivos de yuca (Manihot esculenta) y millo (Sorghum bicolor), entre otros. Además de cría de ganado vacuno y porcino a baja escala, los cuales esporádicamente penetran a la reserva (observación personal). El área presenta pocos musgos y helechos, así como aráceas de los géneros Philodendron y Anthurium, característicos de los arroyos en bosques secos. También se observó una ocurrencia elevada de Spondias mombim, Hura crepitans, Bursera simaruba, Pseudobombax septenatum, Sterculia apétala, Enterolobium cyclocarpum, Aspidosperma polyneuron, Cavanillesia platanifolia, Ochroma pyramidale, Capparis baducca, C.odoratissima y C. indica (Martínez et al. 2010).

\section{Diseño de muestreo}

El estudio se llevo a cabo entre junio y septiembre de 2008. Se realizaron en total cuatro muestreos, a razón de uno por mes. Para la selección de los excrementos se realizó una visita previa a la RCTA, donde se observó que los mamíferos domesticados con mayor presencia en los senderos que bordean el parche de bosque fueron la vaca (B. taurus) y el cerdo (S. domestica), mientras que el mamífero nativo más representativo fue el mono aullador $(A$. seniculus), con una población de $\sim 30$ individuos.

La captura de los escarabajos se realizó mediante trampas de caída, consistentes en un vaso plástico de $0.5 \mathrm{~L}$ que contenía en su interior un tercio de su volumen con una solución de agua con sal, detergente sin olor y alcohol etílico al $70 \%$. En la parte superior se le adaptó un alambre en forma de L invertida, en cuyo extremo se colocó $\sim 20 \mathrm{~g}$ de excremento fresco envuelto en gasa, obtenido de deposiciones frescas de los mamíferos mencionados. En el fragmento se marcaron diez puntos distanciados entre sí $50 \mathrm{~m}$ y en cada uno se colocaron tres trampas de caída en posición triangular distanciadas $20 \mathrm{~m}$ una de otra. Una trampa fue cebada con excremento de vaca, otra con excremento de mono y la tercera con excremento de cerdo. Las trampas fueron retiradas a las 24 horas. La poca distancia entre estas trampas se debió a que muchos de los mamíferos en los fragmentos de Bs-T dejan su excremento en lugares puntuales como quebradas y árboles que proporcionan sombra y refugio. Además, Filgueiras et al. (2009) recomiendan colocar las trampas lo más cerca 
posible en aquellos estudios en los que se desea determinar la preferencia de un recurso por escarabajos coprófagos.

Los individuos recolectados fueron determinados hasta género mediante las claves taxonómicas de Medina \& Lopera (2000) y Vaz de Melo et al (2011). Para especie, se utilizaron las propuestas por Howden \& Young (1981), Edmonds (1994), Kohlmann \& Solís (1997, 2001), Solís \& Kohlmann (2002, 2004), Genier (2009), Escobar (2009) y González et al. (2009). Las determinaciones fueron corroboradas con la colección entomológica de la Universidad del Atlántico (CEUA- UARC) y del Instituto de Investigación de Recursos Biológicos, Alexander von Humboldt (IavH).

\section{Análisis de datos}

La riqueza de especies $(S)$ se tomó como el número de especies capturadas (por trampa, tipo de cebo y muestreos) y la abundancia como el número de individuos. La representatividad del muestreo en el área de estudio se evaluó mediante las curvas de acumulación de especies, utilizando los datos de especies observadas y los estimadores no paramétricos, ICE y Chao 2 por cebo. Los cálculos se realizaron con el programa EstimateS 8.2 (Colwell 2009). La disimilaridad de especies entre cebos se determinó utilizando el índice complementariedad (IC) propuesto por Colwell y Coddington (1994). Para determinar las diferencias en la estructura de la comunidad de Scarabaeinae entre los cebos se aplicó la técnica de ANOSIM (Análisis de Similaridad), con diseño a una vía. Previamente, los datos de abundancia se transformaron a logaritmo $\ln (x+1)$ para contrarrestar el peso de las especies más dominantes, pero sin disminuir su importancia (Clarke \& Warwick 2001) y luego fueron ordenadas en una matriz con base en el índice de similaridad de Bray-Curtis. Considerando las especies que aportaron más de $10 \%$ de la abundancia total se determinó las especies que tipificaron o caracterizaron los cebos mediante la técnica SIMPER (paquete estadístico PRIMER

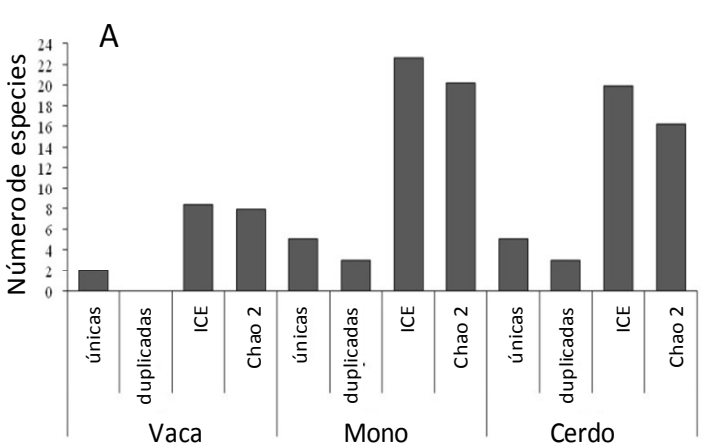

6.0) (Clarke \& Warwick 2001). Los Scarabaeinae capturados fueron separados según los hábitos de relocalización del alimento (i.e., cavadores, rodadores y residentes) de acuerdo a lo propuesto por Hanski \& Camberfort (1991). Para determinar la preferencia por un determinado excremento se utilizó el índice de Manly ( $\alpha$ ), que evidencia el grado de selectividad hacia las presas o dieta consumidas, bajo el criterio de Manly et al. (1993). Si el valor del índice es mayor que $(1 / C$, donde C es la cantidad de cebos utilizados) prefiere, si es menor evita. Este índice fue calculado con el programa HaviStat 1.0 (Montenegro \& Acosta 2008).

\section{RESUltados}

Se capturaron 864 individuos representados en nueve géneros y 21 especies (Tabla 1). Las especies más abundantes fueron Uroxys sp., con 287 individuos, y Uroxys deavilai Delgado \& Kohlmann 2007, con 246 individuos (Tabla 1). Las de menor abundancia fueron Canthon subhyalinus Harold 1867, Eurysternus mexicanus Harold 1869, E. plebejus Harold 1880, Phanaeus hermes Harold 1868 y Canthidium centrale Boucomont 1928, con un individuo cada uno de ellos (Tabla 1).

Según los estimadores no paramétricos ICE (27 especies) y Chao 2 (24 especies), se capturó entre $77.44 \%$ y $87.5 \%$ de las especies de coleópteros coprófagos esperados para el área de estudio (Figura 1A). A nivel de cebos, los valores más bajos de especies capturadas para los estimadores ICE (8.4 especies $=83.33 \%)$ y Chao $2(8$ especies $=88.60 \%)$ fueron para vaca (especies observadas $=7$ ). Mientras tanto, para el mono (especies observadas=18) se presentaron los mayores valores de especies esperadas para el estimador ICE $(23=79.30 \%)$ y Chao $2(20=88.9 \%)$ (Figura 1B). Los resultados de la curva de acumulación a nivel de cebos

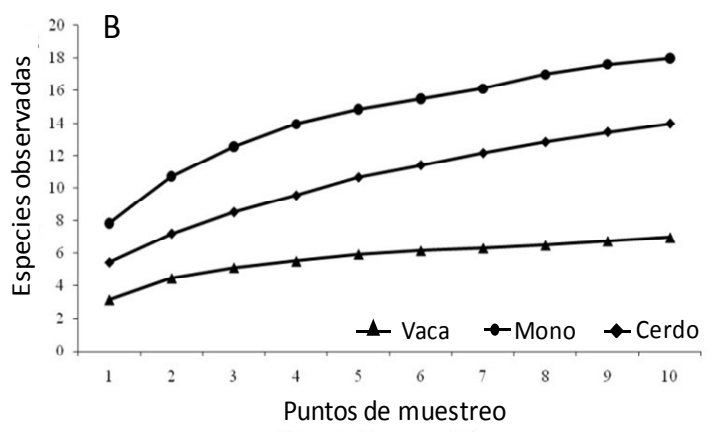

Figura 1. Valor de los estimadores (A) y curva de acumulación de especies observadas (B) para los tres excrementos en el área estudio.

Figure 1. Value of the estimators for the types of baits in the study area (A) and species accumulation curve observed (B) for bait in the area of study. 
Tabla 1. Riqueza y abundancia de Scarabaeinae (Scarabaeidae) por cebo, en la RCTA, Atlántico, Colombia. Abreviaturas: Índice de Manly Alpha ( $\alpha$ i) Vaca (V), Mono (M), Cerdo (C); Cavador (Cav), Rodador (Rod), Residente (Res); especies únicas para vaca $(*)$, mono $(* *)$ y cerdo $(* * *)$.

Table 1. Variation in the richness and abundance of dung beetles (Scarabaeidae: Scarabaeinae) for bait, from the Rural Reserve of Tierra Arena (RCTA), Atlántico, Colombia. Abbreviations: Manly Alpha Index ( $\alpha$ i) Cow (V), Monkey (M), Pig (C), Tunneler (Cav), Roller (Rod), Dweller (Res); unique species for cow $(*)$, monkey $(* *)$ and pig $(* * *)$.

\begin{tabular}{|c|c|c|c|c|c|c|c|c|}
\hline \multirow[b]{2}{*}{ Especies } & \multicolumn{3}{|c|}{ Tierra Arena } & \multirow[b]{2}{*}{ Total } & \multicolumn{3}{|c|}{ Manly Alpha ( $\alpha \mathrm{i})$} & \multirow[b]{2}{*}{ Gremio } \\
\hline & $\mathrm{V}$ & $\mathrm{M}$ & $\mathrm{C}$ & & $\mathrm{V}$ & $\mathrm{M}$ & $\mathrm{C}$ & \\
\hline Dichotomius belus** & 0 & 2 & 0 & 2 & 0 & 1 & 0 & Cav \\
\hline Deltochilum sp. & 0 & 2 & 0 & 2 & 0 & 1 & 0 & Rod \\
\hline Uroxys sp. & 23 & 168 & 96 & 287 & 0.2 & 0.5 & 0.4 & Cav \\
\hline U. deavilai & 26 & 134 & 86 & 246 & 0.2 & 0.4 & 0.4 & Cav \\
\hline U. micros & 0 & 33 & 6 & 39 & 0 & 0.8 & 0.2 & Cav \\
\hline Canthon aff. morsei & 6 & 106 & 52 & 164 & 0.1 & 0.6 & 0.4 & Rod \\
\hline C. cyanellus & 6 & 14 & 23 & 43 & 0.3 & 0.2 & 0.5 & Rod \\
\hline C. juvencus ${ }^{* *}$ & 0 & 3 & 0 & 3 & 0 & 1 & 0 & Rod \\
\hline C. subhyalinus ${ }^{* *}$ & 0 & 1 & 0 & 1 & 0 & 1 & 0 & Rod \\
\hline C. septemmaculatus & 0 & 1 & 1 & 2 & 0 & 0.4 & 0.6 & Rod \\
\hline Scybalocanthon $\mathrm{sp}^{* *}$ & 0 & 2 & 0 & 2 & 0 & 1 & 0 & Rod \\
\hline Eurysternus plebejus ${ }^{* * *}$ & 0 & 0 & 1 & 1 & 0 & 0 & 1 & Res \\
\hline E. mexicanus ${ }^{* * *}$ & 0 & 0 & 1 & 1 & 0 & 0 & 1 & Res \\
\hline Onthophagus sp & 1 & 6 & 1 & 8 & 0.3 & 0.6 & 0.1 & Cav \\
\hline O. acuminatus & 1 & 12 & 2 & 15 & 0.2 & 0.7 & 0.2 & Cav \\
\hline O. clipeatus & 0 & 2 & 2 & 4 & 0 & 0.4 & 0.6 & Cav \\
\hline O. marginicollis & 13 & 6 & 14 & 33 & 0.6 & 0.1 & 0.3 & Cav \\
\hline Phanaeus hermes** & 0 & 1 & 0 & 1 & 0 & 1 & 0 & Cav \\
\hline Canthidium $\mathrm{sp}$ & 0 & 6 & 2 & 8 & 0 & 0.7 & 0.3 & Cav \\
\hline C. centrale ${ }^{* *}$ & 0 & 1 & 0 & 1 & 0 & 1 & 0 & Cav \\
\hline C. haroldi $i^{* * *}$ & 0 & 0 & 2 & 2 & 0 & 0 & 1 & Cav \\
\hline Riqueza (S) & 7 & 18 & 14 & 21 & & & & \\
\hline Abundancia (N) & 76 & 499 & 289 & 864 & & & & \\
\hline
\end{tabular}

muestran una estabilización del 100\% de las especies asociadas al excremento de vaca y del $75 \%$ para mono (Figura $1 \mathrm{~B}$ ).

El valor más alto de riqueza (18 especies) y abundancia (499 individuos) se presentó en las trampas cebadas con excremento de mono (Tabla 1). El excremento de vaca no presentó especies exclusivas. Dichotomius belus, Deltochilum sp., Canthon juvencus, C. subhyalinus, Scybalocanthon sp., P. hermes y C. centrale fueron exclusivas para el excremento de mono y E. plebejus, E. mexicanus y Canthidium haroldi para el de cerdo (Tabla 1). Se compartieron siete especies (33.33\%) entre los tres cebos. El valor más alto del Índice de Complementariedad (IC) se presentó entre el excremento de vaca-mono $(\mathrm{IC}=0.61)$. Le siguió el de vaca-cerdo $(\mathrm{IC}=0.50) \mathrm{y}$, por último, el de mono-cerdo, con un $\mathrm{IC}=0.48$, indicando un mayor número de especies compartidas (11).

La prueba de ANOSIM indicó diferencias en la composición y la estructura de la comunidad de Scarabaeinae entre los tres cebos $(R=0.42$; $p=0.001)$. La mayor similaridad seobservó entre el excremento mono-cerdo $(\mathrm{R}=0.068 ; p=0.091)$ y la menor entre vaca-mono $(\mathrm{R}=0.68 ; p=0.001)$. La rutina de similaridad SIMPER demostró que las especies $U$. deavilai y $U$. sp. tipifican los tres cebos (Tabla 2). Los excrementos de mono y cerdo fueron caracterizados por Canthon aff. morsei Howden 1966, mientras que el de vaca fue tipificado por Onthophagus marginicollis Harold 1880 (Tabla 3). El mayor porcentaje de similaridad (valor promedio $=67.50 \%$ ) se presentó en el excremento de cerdo debido al aporte que hacen a la abundancia las especies U. deavilai, U. sp., C. aff. morsei y C. cyanellus (Tabla 2).

Tabla 2. Porcentajes de similaridad (SIMPER) de especies que caracterizan a cada uno de los excrementos en la RCTA, Atlántico, Colombia.

Table 2. Similarity percentages (SIMPER) of species that characterize each of the baits from the RCTA, Atlántico, Colombia.

\begin{tabular}{lccc}
\hline \multirow{2}{*}{ Especies/Cebo } & \multicolumn{3}{c}{ Contribución (\%) } \\
& Vaca & Mono & Cerdo \\
\hline Uroxys deavilai & 38.12 & 21.66 & 33.13 \\
U. sp. & 32.17 & 28.74 & 30.07 \\
Onthophagus marginicollis & 27.12 & & \\
Canthon aff. morsei & & 24.57 & 19.48 \\
C. cyanellus & & & 12 \\
Acumulado (\%) & 97.4 & 74.98 & 94.69 \\
Similaridad promedio (\%) & 60.03 & 56.37 & 67.5 \\
\hline
\end{tabular}




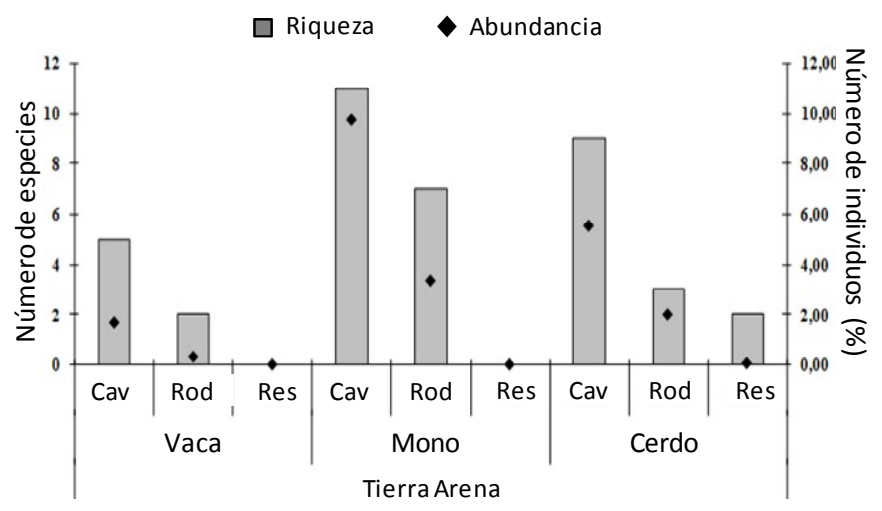

Figura 2. Riqueza y abundancia (\%) de los gremios funcionales de Scarabaeinae en la RCTA.

Figure 2. Structure of functional guilds of dung beetles from the RCTA (abundance values are given in percentage).
La mayor riqueza y abundancia fue de las especies cavadoras sobre las rodadoras y las residentes, independientemente del tipo de cebo utilizado (Figura 2). La mayor riqueza y abundancia de cavadores y rodadores se capturó en las trampas cebadas con excremento de $A$. seniculus, mientras que los pocos especímenes de residentes sólo cayeron con excremento de cerdo (Figura 2). El índice de Manly $(\alpha)$ indicó que la mayoría de las especies recolectadas (13) en la localidad tuvieron mayor preferencia por el excremento de mono, seguido por el de cerdo con seis y para vaca con una especie (Tabla 1).

\section{DISCUSIÓN}

Este estudio constituye uno de los primeros esfuerzos para el conocimiento de las preferencias alimentarias de los escarabajos coprófagos en fragmentos de Bs-T en las zonas bajas del Caribe colombiano. Los resultados sugieren que la introducción de mamíferos exóticos a áreas bien conservadas de bosque seco debido a la expansión de la frontera agrícola-ganadera puede afectar de manera indirecta y negativa a los ensambles de escarabajos coprófagos.

Al comparar el número de especies encontradas con las registradas para la región Caribe por Escobar (1998), Martínez et al. (2010) y Barraza et al. (2010), se evidencia una representatividad de $\sim 75.5 \%$. Lo anterior indica que el área de estudio mantiene una fauna representativa de escarabajos coprófagos del Bs-T de las tierras bajas del Caribe colombiano. También se demuestra que cuando se utilizan diferentes tipos de excrementos como atrayente en la misma zona, las oportunidades de capturar un mayor número de especies aumenta (Martin-Piera \& Lobo 1996).
Según los estimadores, se capturó la mayoría de las especies esperadas tanto para el área de estudio como para cada excremento. El menor número de especies compartidas entre excremento de vaca-mono posiblemente obedezca a que los Scarabaeinae muestren cierta preferencia por el excremento de mono por ser un mamífero nativo de la reserva (Gill 1991). Además, el excremento de vaca no se encuentra habitualmente en la reserva al tiempo que los escarabajos coprófagos del interior del bosque posiblemente tengan poca afinidad por el estiércol del ganado. Por otro lado, a pesar de que el excremento de cerdo no es de un mamífero nativo y que se encuentra en las afueras de la reserva, el cerdo es omnívoro, y esto hace más atractivo al excremento para los escarabajos coprófagos debido a la concentración elevada de nitrógeno y fosforo, nutrientes fundamentales para que los adultos inmaduros finalicen el desarrollo de su sistema muscular y las hembras culminen la maduración de sus huevos (Hanski 1991). También, parte importante de la dieta de los Scarabaeinae la constituyen las bacterias que se encuentran en gran cantidad en el excremento de omnívoros. Estas bacterias son fundamentales para el metabolismo de estos insectos (Bustos-Gómez \& Lopera 2003).

La tipificación de los tres cebos por $U$. deavilai y la morfoespecie $U$. sp., se atribuye a que estos escarabajos pueden aprovechar y/ o adaptarse a cualquier tipo de excremento, mostrando un comportamiento trófico generalista. Esto también demuestra que las especies más abundantes no presentan en la mayoría de los casos un comportamiento de especialización en la dieta (Filgueiras et al. 2009). La alta incidencia de O. marginicollis en las trampas con excremento de vaca se puede atribuir a que este escarabajo tiene preferencia por espacios abiertos, borde y pastizales 
(Delgado \& Gil 1998; Bustos-Gómez \& Lopera 2003), donde predomina una alta biomasa del excremento de mamíferos introducidos. Por su parte, $C$. aff. morsei tiene preferencia por zonas del interior de fragmentos de Bs-T, donde la disponibilidad de excremento no es muy abundante y existe una intensa competencia y una especialización por un determinado recurso (Hanski 1991; Hanski \& Camberfort 1991) como el excremento de mono. La mayor similaridad en el excremento de cerdo se debe al aporte que hacen a la abundancia las especies más representativas del muestreo, lo que se puede atribuir a sus características omnívoras.

A nivel de relocalización del alimento, la dominancia de las especies cavadoras puede estar relacionada con las características del suelo (limoso-arcilloso y arcillo-arenoso) en el área de estudio, que favorece el establecimiento de nidos en galerías para este gremio funcional y facilita que entierren el alimento más rápidamente, evitando la mortalidad de adultos y larvas (Hanski \& Camberfort 1991; Martínez et al. 2010). Además, la predominancia del gremio cavador cobra gran importancia en este fragmento de bosque debido a que este grupo de escarabajos tiene mayor capacidad de procesar materia fecal que los escarabajos rodadores de tamaño similar (Doube 1990) dada la cercanía de sus túneles a la fuente del excremento (Andresen 2005). Estos resultados son similares a los obtenidos por Barraza et al. (2010) en un paisaje de bosque seco tropical en Bahía Concha (Santa Marta), donde observó una proporción de 1.2: 1 de cavadores frente a rodadores y una muy baja proporción de residentes.

La mayor riqueza y abundancia de cavadores y rodadores en las trampas con excremento de A. seniculus se puede atribuir a la capacidad que tiene un determinado gremio de escarabajos en manipular uno $\mathrm{u}$ otro tipo de heces teniendo en cuenta su textura; esto facilita que algunos escarabajos vivan en el interior o construyan galerías debajo del excremento o que puedan rodar la bola-nido más fácilmente (Lobo 1985). Además, la mayoría de las veces el excremento de este primate cae desde arboles de gran altura y queda muy disperso en el suelo, facilitando la actividad de la especies rodadoras. La baja proporción del gremio de residentes puede tener mucha relación con la cantidad de excremento que estos requieren (Halffter \& Edmonds 1982) debido a que realizan sus galerías al interior del mismo excremento, por lo que podrían tener más éxito cuando el recurso es más abundante (Howden \& Young 1981).

Según los resultados del índice de Manly, en la RCTA las especies de Scarabaeinae muestran una tendencia a preferir un tipo de excremento. Sin embargo, estos resultados podrían cambiar debido a que las preferencias pueden variar en el tiempo y en el espacio en función de las interacciones ambienteestiércol (Martin-Piera \& Lobo 1996), en especial, en lugares donde las condiciones climáticas son muy cambiantes como el Bs-T (Escobar 1998). Los resultados de este estudio difieren de lo reportado por Bustos-Gómez \& Lopera (2003) y de la hipótesis de Halffter \& Matthews (1966), quienes asumen que la generalización de la dieta es una característica de las comunidades de escarabajos coprófagos neotropicales. Además, la alta preferencia por el excremento de mono permite inferir que a pesar de que existan otras fuentes de excremento ocasionales (i.e., excrementos de vaca y cerdo), las especies de escarabajos coprófagos prefieren (ciertas especies) o dependen (otras especies) del recurso que los mamíferos nativos proveen. Sin embargo, la mayoría de los escarabajos coprófagos que no muestran preferencia en este estudio estuvieron representadas por muy pocos ejemplares, por lo cual, la no-preferencia de estas especies por un excremento puede deberse al tamaño limitado de la muestra (Dormont et al. 2004).

Finalmente, este estudio demuestra la importancia del excremento de los primates como recurso alimenticio en los fragmentos Bs-T (Anzures-Dadda et al. 1998). Además, se demuestra que los fragmentos de Bs-T en la región Caribe no sólo albergan una fauna representativa de Scarabaeinae sino que además presentan relaciones estrechas entre mamíferos nativos e insectos. El avance de pasturas sobre fragmentos de bosque seco y el eventual reemplazo de los primates por mamíferos exóticos podría afectar el ensamble de escarabajos coprófagos, particularmente reduciendo las poblaciones que en este estudio mostraron marcadas preferencias por el excremento de mono. Por consiguiente, se hace necesario realizar estrategias para la protección de la fauna reduciendo el empobrecimiento de hábitat y la presión antrópica sobre el bosque seco tropical. 
Agradecimientos: Agradecemos a la comunidad campesina de Tierra Arena, por su colaboración durante la realización del trabajo. A los integrantes del Semillero de Investigación de Insectos NEOPTERA del programa de Biología de la Universidad del Atlántico, por su colaboración en las faenas de campo y a los evaluadores anónimos quienes con sus observaciones contribuyeron a mejorar y enriquecer este documento.

\section{BIBLIOGRAFÍA}

ANDRESEN, E. 2005. Interacción entre primates, semillas y escarabajos coprófagos en bosques húmedos tropicales: un caso de diplocoria. Universidad y Ciencia, número especial, II:73-84.

Anzures-Dadda, A; A Estrada \& R Coates-Estrada. 1998. Monos aulladores (Alouatta palliata), escarabajos coprófagos y la fragmentación de las selvas en los Tuxtlas, Veracruz, México. Neotropical Primates, 6:111-114.

Barraza, J; J Montes; N Martínez \& C Deloya. 2010. Ensamblaje de escarabajos coprófagos (Scarabaeidae: Scarabaeinae) del Bosque Tropical Seco, Bahía Concha, Santa Marta, Colombia. Revista Colombiana de Entomología, 36:285- 291.

BoHórquez, J \& J Montoya. 2009. Abundancia y preferencia trófica de Dichotomius belus (Coleóptera: Scarabaeidae) en la reserva forestal de Colosó, Sucre. Boletín del Museo de Entomología de la Universidad del Valle, 10:1-7.

Bustos-Gómez, F \& A Lopera. 2003. Preferencia por cebo de los escarabajos coprófagos (Coleóptera: Scarabaeidae: Scarabaeinae) en un remanente de bosque seco tropical al norte del Tolima (Colombia). M3m monografías tercer milenio vol. 3, SEA, Zaragoza, 30:59-65.

CASTEllanos, M; FEscobar \& PSTEVENSON. 1999. Dung Beetles (Scarabaeidae: Scarabaeinae) Attracted to Woolly Monkey (Lagothrix lagothricha Humboldt) Dung at Tinigua Park, Colombia. The Coleopterist Bulletin, 53:155-159.

Clarke, KR \& RM WarWick. 2001. Change in marine communities: An approach to stadistical analysis and interpretation. Natural Environment Research Council, UK. Pp. 144.

Colwell, RK \& JA Coddington.1994. Estimating terrestrial biodiversity through extrapolation. Philosophical Transactions of the Royal Society (Series B), 345:101-118.

Colwell, RK. 2009. Estimates: Statistical Estimation of Species Richness and Shared Species from Samples, Version 8.2. En viceroy.eeb.uconn.edu/estimates.

DeLGado, LCM \& PZN GIL. 1998. Estudio de las comunidades de insectos con énfasis en los escarabajos copronecrófagos (Coleoptera: Scarabaeidae) en cuatro estados sucesionales en la zona de in uencia al proyecto hidroeléctrico Porce II. Tesis de grado (Ingeniero Agrónomo) Universidad Nacional de Colombia. Pp. 175.

Dormont, L; G Epinat \& JP Lumaret. 2004. Trophic Preferences Mediated by Olfactory Cues in Dung Beetles Colonizing Cattle and Horse Dung. Environmental Entomology, 33:370-377.

DoubE, BM. 1990. A functional classification analysis of the structure of dung beetle assemblages. Ecological Entomology, 15:371-383.

Edmonds, WD. 1994. Revision of Phanaeus Macleay, a New World Genus of Scarabaeinae Dung Beetles (Coleoptera: Scarabaeidae, Scarabaeinae). Contributions in Science Natural History Museum of Los Angeles Country, 443:1-105.
Escobar, F. 1998. Análisis regional de la comunidad de escarabajos coprófagos (Coleóptera: Scarabaeidae: Scarabaeinae) de los Bosques Secos de la región Caribe de Colombia. Pp. 72-75 en: Chávez, ME \& N Arango (eds.). Informe Nacional del Estado de la Biodiversidad. Colombia, Tomo I: Diversidad Biológica. Instituto Humboldt, PNUMA y Ministerio del Medio Ambiente. Bogotá. Pp. 535.

Escobar, F; JM Lobo \& G Halffter. 2005. Altitudinal variation of dung beetle (Scarabaeidae: Scarabaeinae) assemblages in Neotropical mountains. Global Ecology and Biogeography, 14:327-337.

Escobar, A. 2009. Taxonomía y variación morfológica del complejo de especies del género Uroxys (Scarabaeidae: Scarabaeinae) en el Sistema Regional de Áreas Protegidas del Eje Cafetero (SIRAP-EC). Trabajo de Pregrado. Universidad del Valle. Facultad de Ciencias. Programa de Biología. Cali, Valle del Cauca, Colombia. Pp. 116.

Estrada, A; G Halffter; R Coates-Estrada \& DAM Junior. 1993. Dung beetles attracted to mammalian herbivore (Alouatta palliata) and omnivore (Nasua narica) dung in the tropical rain forest of Los Tuxtlas, México. Journal of Tropical Ecology, 9:45-54.

Filgueiras, B; C Liberal; C Aguiar; CM Medina \& L IANNUZZI. 2009. Attractivity of omnivore, carnivore and herbivore mammalian dung to Scarabaeinae(Coleóptera, Scarabaeidae) in a tropical Atlantic rainforest remnant. Revista Brasileira de Entomología, 53:422-427.

Genier, F. 2009. Le genre Eurysternus Dalman, 1824 (Scarabaeidae: Scarabaeinae: Oniticellini), revision taxonomique et cles de determination illustrees. SofiaMoscow.

GILL, BD. 1991. Dung beetles in tropical American forests. Pp. 211-229 en: Hanski, I \& Y Camberfort (eds.). Dung Beetle Ecology. Princeton, New Jersey. USA.

GonZÁLEZ, FA; F Molano \& CA Medina. 2009. Los subgéneros Calhyboma, Hybomidium y Telhyboma (Coleoptera: Scarabaeidae: Scarabaeinae: Deltochilum) en Colombia. Revista Colombiana de Entomología, 35:253-274.

Halffter, G \& EG Matthews. 1966. Natural history of dung beetles of the subfamily Scarabaeinae (Coleoptera: Scarabaeidae). Folia Entomológica Mexicana, 12-14:1-312.

HalfFTER, G \& WD EDMONDS. 1982. The nesting behavior of bung beetle (Scarabaeinae) Instituto de Ecología, México.

Hanski, I. 1991. The dung insect community. Pp. 5-21 en: Hanski, I \& Y Camberfort (eds.). Dung Beetle Ecology. Princeton, New Jersey.

Hanski, I \& Y Cambefort. 1991. Dung Beetle Ecology. Princeton University Press, New Jersey.

Hernández, J; A Hurtado; R Ortiz \& T Walschburger. 1991. Unidades Biogeográficas de Colombia. En: La Diversidad Biológica de Iberoamérica I. CYTED-B Programa Iberoamericano de Ciencia y Tecnología para el Desarrollo, México.

Holdridge, LR; WC GrenKe; WH HatheWAy; T Liang \& JA Tosi. 1971. Forest environments in tropical life zones, a pilot study. Pergamon Press, Oxford.

Howden, H. \& O Young. 1981. Panamanian Scarabaeinae: taxonomy, distribution and habits (Coleóptera: Scarabaeidae). Contributions of the American Entomological Institute, 18:1-204.

Instituto de Investigación de Recursos Biológicos Alexander von Humboldt- IAvH. 1998. El Bosque seco Tropical (Bs-T) en Colombia. Grupo de Exploraciones 
y Monitoreo Ambiental GEMA, IavH, Villa de leyva Colombia.

Kohlmann, B \& A Solís. 1997. El género Dichotomius (Coleoptera: Scarabaeidae) en Costa Rica. Giornale Italiano di Entomología, 8:343-382.

Kohlmann, B \& A Solís. 2001. El género Onthophagus (Coleoptera: Scarabaeidae) en Costa Rica. Giornale Italiano di Entomología, 9:159-261.

Lово, JM. 1985. Algunos datos y observaciones sobre la influencia del origen del excremento en las estructuras de las comunidades de Scarabaeoidea (Col.) coprófagos. Boletim da Sociedade Portuguesa de Entomologia, 3(I):45-55.

Manly, B; L Mcdonald \& D Thomas. 1993. Resource Selection by Animals, Statistical Design and Analysis for Field Studies. Chapman \& Hall. London, Pp. 10.

Martín-Piera, F \& JM Lobo. 1996. A comparative discussion of trophic preferences in dung beetles communities. Miscellania Zoologica, 19:13-31.

Martínez, N; L Cañas; J Rangel; J Barraza; J Montes; ET AL. 2010. Coleópteros Coprófagos (Scarabaeidae: Scarabaeinae) en un Fragmento de Bosque Seco Tropical en el Departamento del Atlántico, Colombia. Boletín del Museo de Entomología de La Universidad del Valle, 11:21-30.
Medina, CA \& A Lopera. 2000. Clave ilustrada para la identificación de géneros de escarabajos coprófagos (Coleóptera: Scarabaeinae) de Colombia. Caldasia, 22: 299-315.

Medina, C; A Lopera; A Vitolo \& B Gill. 2001. Escarabajos Coprófagos (Coleóptero: Scarabaeidae: Scarabaeinae) de Colombia. Biota Colombiana, 2:131-144.

Montenegro, J \& A Acosta. 2008. Programa innovador para evaluar uso y preferencia de hábitat. Universitas Scientiarum, 13:208-217.

Mora-Aguilar, EF \& E Montes De Oca. 2009. Escarabajos necrófagos (Coleoptera: Scarabaeidae y Trogidae) de la región central baja de Veracruz, México. Acta Zoológica Mexicana (n.s.), 25:569-588.

Solís, A \& B Kohlmann. 2002. El género Canthon (Coleoptera: Scarabaeidae) en Costa Rica. Giornale Italiano di Entomologia, 10:1-68.

Solís, A \& B Kohlmann. 2004. El género Canthidium (Coleoptera: Scarabaeidae) en Costa Rica. Giornale Italiano di Entomologia, 11:1-73.

Vaz de Mello, F; WD Edmonds; FC Ocampo \& P SCHOOLMEESTERS. 2011. A multilingual key to the genera and subgenera of the subfamily Scarabaeinae of the New World (Coleoptera: Scarabaeidae). Zootaxa, 2854:1-73. 